\title{
A decision support system with EDA_PR algorithm for the hot rolling scheduling
}

\author{
Xiaoxia Zhang \\ College of Software Engineering \\ University of Science and Technology Liaoning \\ Anshan 114051, P.R.China \\ aszhangxx@163.com
}

\author{
Yunyong Ma \\ College of Software Engineering \\ University of Science and Technology Liaoning \\ Anshan 114051, P.R.China \\ 1059293613@qq.com
}

\begin{abstract}
This paper presents a hybrid algorithm for the hot rolling scheduling problem, which is derived from the actual steel production, and some features make the solution methodology more difficult. The hybrid strategy is based on the solution construction mechanism of estimation of distribution algorithm (EDA) with path relinking (PR), an evolutionary method, which results in a novel approach that we call EDA_PR. Moreover, a decision support system in which the algorithm has been embedded for the hot rolling scheduling is designed. The computational experiments show that the EDA_PR method has more potential for improvement to solve the hot rolling scheduling problem compared with the manual scheduling method.
\end{abstract}

Keywords- Estimation of distribution algorithm; path relinking; hot rolling scheduling; the decision support system.

\section{INTRODUCTION}

The modern iron and steel industries are beginning to give more serious attention to the theoretical and technical methods that promise to increase steel production efficiency and make the best of their existing capacities to ensure their survival in the global market. In the hot strip mill, a typical problem is how to make the rolling scheduler, which plays an important role not only in the hot strip mill, but also in the integrated production of continuous casting and hot-charging rolling.

Most steel production facilities produce a variety of finished products according to customer orders. Each order requires the production of a number of coils with the required physical properties and dimensions, and each coil corresponds to one slab. Raw materials are transformed by a series of chemical reactions in the blast furnace into liquid iron [1]. Next, the liquid iron is transferred into liquid steel through chemistry and purification processes in the melt shop. The hot strip mill transfers steel slabs into steel coils by subjecting the slabs first to high temperatures in a furnace and then to high pressures through a series of rolls. Because of suffering the high temperature, high speed, heavy wear and tear, work rollers and backup rollers on each stand often need to be replaced at regular intervals. In hot strip mill production, roller performance plays a significant role in determining product quality and setup cost. Roller replacements involve a very high setup cost, so we wish to replace rollers as infrequently as possible [2,3], while still maintaining the high coil quality.
We investigate the hot rolling scheduling problem for the actual steel production. The existing manual scheduling systems use a serial strategy and heavily rely on skilled human experts. Therefore the scheduling horizon was limited to their experiences. In order to make the automated scheduler more efficiently, we adopted a parallel strategy here to simultaneously group the slabs into multiple turns. The scheduling objective is to find the effective product scheduling that minimizes the costs of roller replacement, total changeover costs between adjacent slabs. Improving product quality requires changing the rollers as frequently as possible, whereas minimizing the costs of roller replacement requires that a work roller should roll as many slabs as possible in a turn. Therefore, there are some tradeoffs between these objectives and they will be obtained by the effective hot rolling scheduling.

The hot rolling scheduling is an important research topic and has been widely explored recently. Although the development of modern heuristics has led to considerable progress in steel rolling mills, the quest for improved performance continues. Balas (1989) [4] presents a prize collecting traveling salesman problem (PCTSP), which is a generalization of the traveling salesman problem (TSP). This is theoretical paper and therefore does not report any real application of his solution methods. Tang et al. [5] modeled the hot rolling scheduling problem as a multiple traveling salesman problem (MTSP) based on actual production constraints.

The paper is organized as follows. Section II introduces methodology. Section III gives the details of decision support system construction. Section IV presents some conclusions

\section{EDA PR ALGORITHM}

Recently, estimation of distribution algorithm (EDA) has attracted much attention by evolutionary algorithm researchers due to their search abilities. Estimation of distribution algorithm is one of the most popular evolutionary algorithms which estimate the probability distribution associated with the selected individuals and sample this distribution to create the next population in recent years [6-7]. Different from the GA that produces offspring through crossover and mutation operations, estimation of distribution algorithm (EDA) does it by sampling according to a probability model which has a great effect on the performances of EDA. It is the key issue how 
to construct the probability model of EDA. EDA constructs promising probabilistic models from superior solutions found thus far, which uncover some important regularities of problems. EDA can efficiently evolve the whole population towards the promising regions of the global optimum. Hence, the pseudo-code of the basic EDA procedure is outlined as Figure 1, where $D_{0}$ indicates the set of initial population and $N$ denotes the superior population according to a fitness function. In order to generate population based on the probability model, we should generate the selection

Procedure Estimation of Distribution Algorithm

begin

Generate and evaluate initial population $D_{0}$

repeat

Select superior population $\mathrm{N}$ from $D_{0}$

Estimate the probability distribution of the superior population

Build the probability model $p(t)$ from $N$

Sample $p(t)$ to generate new population

Until Stopping condition is met

End

Figure 1. Pseudo-code of the basic EDA

probability matrix. Let $p(t)$ be the probabilistic matrix of all nods at different positions, they are defined as the following probability matrix:

$$
p(t)=\left(\begin{array}{cc}
p_{11}(t) & \ldots . . p_{1 n}(t) \\
\ldots & \\
p_{n 1}(t) & \ldots p_{n n}(t)
\end{array}\right)
$$

where $p_{i j}(t)$ is the selection probability of position $j$ at every position $i$, and $t$ denotes at current generation. The key point of EDA is how the probability distribution is estimated at each generation. Our probability model is similar to PBIL where the $p_{i j}(t)$ is updated as follows:

$$
\begin{aligned}
p_{i j}(t) & =\left(\begin{array}{r}
(1-\alpha) \frac{1}{L}+\frac{1}{n} \sum_{k=1}^{n} \delta_{i j}\left(x_{k}^{0}\right), \quad i \neq j \\
0
\end{array}\right. \\
& \text { Where } i=1,2, \ldots n, j=1,2, \ldots n .
\end{aligned}
$$

Assuming that $\delta$ denote a feasible solution, and the sequence of nodes is $(\delta(1), \delta(2), \ldots, \delta(n))$ where $\delta(i)(i=$ $1,2, \ldots, n)$ represents the node that has been visited at the ith node, and they are defined as the eq.(3).

$$
\delta_{i j}\left(\pi_{k}^{0}\right)=\left(\begin{array}{c}
1, \pi_{k}^{0}(l)=i^{\wedge} \pi_{k}^{0}((\bmod L)+1)=j \\
0
\end{array}\right.
$$

As described in the figure, the main calculation procedure of the basic EDA is that (1) firstly, the $N$ selected individuals are selected from the population in the previous generation. (2) Secondly, the probabilistic model is estimated from the genetic information of the selected individuals. (3) A new population is then sampled by using the estimated probabilistic model. (4) Finally, the new population is evaluated. (5) Steps (1)-(4) are repeated until the termination criterion is satisfied. EDA has its own strength and weakness. Therefore, much research has tried to develop the quest for the performance of hybrid algorithms expecting to achieve the effectiveness and efficiency. The hybrid algorithm includes the two basic steps of construction of solutions and improvement strategies of the solution, when it was adapted to solve the hot rolling scheduling problem.

The central concept behind Path-relinking, as applied to for hot rolling scheduling problem, is the attempt to improve the total cost of turns by transferring small slabs. Path relinking (PR) is a novel evolutionary method, and it was introduced by Glover and Laguna [8] for integrating diversification and intensification in tabu search. This approach generates new solutions by exploring trajectories that connect high quality solutions in a reference set RefSet. The reference set RefSet is a set of feasible solutions, and it consists of a total of high quality solutions ( RefSet $\left._{1}\right)$ and diverse solutions $\left(\operatorname{RefSet}_{2}\right)$. Each trajectory starts from one of the solutions, called an initial solution, and leads toward the other solution, called a guiding solution. This trajectory is accomplished by performing moves on the current solution, such that attributes in the guiding solution are gradually introduced, and attributes not in the guiding solution are increasingly dropped. Identical parts of two solutions should remain unchanged during the process. Unlike genetic algorithms, where two solutions are randomly chosen from the population to generate new solutions, path relinking utilizes systematic, deterministic rules for combining solutions [9]. During the path relinking phase, a series of intermediate solutions are generated by moving away from the initiating solution. These solutions contain fewer attributes from the initiating solution and more from the guiding solution as the current solution moves along the trajectory. The rekinked path (trajectory) may encounter intermediate solutions that may be better than the initiating or guiding solution. The PR procedure is outlined as Figure 2 .

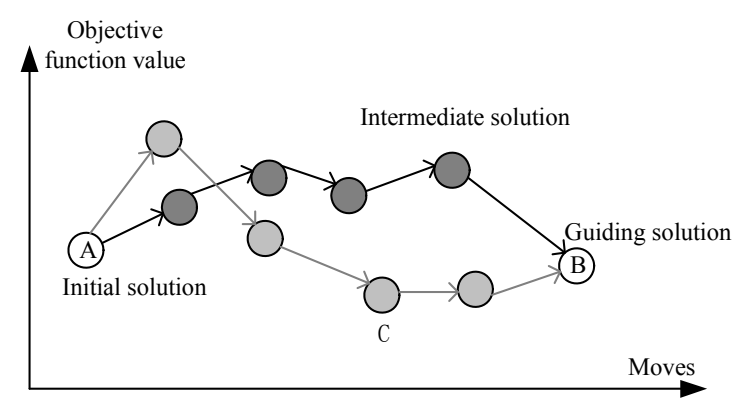

Figure 2. Process flow of path relinking.

The EDA_PR algorithm is proposed in this paper, which is to hybridize EDA and PR with heuristics to solve the hot rolling scheduling problem. The method has both the advantages of EDA, the ability to find the higher performance solutions, and that of PR, the ability to conduct fine-tuning in the quality of solutions and to find better solutions. Meanwhile, PR is embedded into EDA as 
neighborhood search to improve solutions. In fact, the slabs in the staple material section cannot be used in the warm up material section. However, in special condition the slabs in the warm up material section can be used in the staple material section and this incurs some costs. And then, in order to obtain higher solution, two heuristics are adopted to see if an overall improvement in the objective function can be attained. 2-opt heuristic is applied at all times to act as an improvement heuristic within the turn, while the PR procedure is used for the between-turn improvement. The better solution obtained from the EDA as an initial parameter is then applied to the PR procedure. The PR procedure receives this parameter and improves it. To save computation time, it isn't necessary to call the PR procedure at every iteration. We restrict the certain frequencies of using the PR procedure, or we call procedure only if the best solution is improved. This method can reduce the computation time requirements to reasonable levels both for computing auxiliary graph arc costs and for searching the auxiliary graph.

\section{DECISION SUPPORT SYSTEM CONSTRUCTION}

For practical constraints influencing the hot rolling scheduling should be taken into account, it is not easy for a human planner to generate multiple turns in very short time spans. It is necessary to design the decision support system for the hot rolling scheduling, which the planner uses to deal with a wide range of possible production scenarios. We have designed a decision support system for the hot rolling scheduling. The system incorporates the mathematical algorithms, modern information technology and expertise in the hot rolling scheduling, so the decision support system not only provides mechanisms to improve planning ability and overall system effectiveness, but also deals with the problem of planning with uncertain information.

\section{A. Architecture of the decision support system}

The decision support system is designed to improve the existing scheduling process according to the actual hot rolling production in China. The model is characterized by some new features. These features make the solution methodology more difficult. Therefore, a hybrid strategy is proposed to determine good approximate solutions for this complicated problem. The emphasis of the designed decision system is that EDA and EDA PR algorithms have been embedded in the designed decision system, as this is the basis for the successful decision system. The system can produce better solutions in the short time available, and the graphical user interface allows the planner to modify easily the solutions manually by analyzing the resulting solutions before accepting the solution for production. The architecture of the decision support system developed for the hot rolling scheduling problem consists of following components: Data collection system, Data access platform, EDA, EDA_PR algorithm and hot rolling scheduling editor.

- Data collection system. The data collection component consisted of the data collection, data retrieving and Data Analyzing. The data collection can download customer's data and production data from the central database as needed. Once the necessary data has been gathered, it can be repeatedly used in the data retrieving procedure at different time points. This enabled scheduler to analyze and manage the data. Data Analyzing can analyze and manage customer's data demanding service and production data as needed.

- Data access platform. The data administrator must update the databases and guarantee the data information safety through the Data access platform. The database used is SQL Server and it can be easily accessed and manipulated through a file component. It is sufficient that the data administrator is able to consult and modify the data as needed.

- EDA_PR algorithm. The hybrid algorithm first constructs a feasible solution for the hot rolling scheduling using EDA procedure then finds an improving neighbor using PR method. The advantage of the algorithms is their ability to handle efficiently a large number of constraints and parameters of the hot rolling scheduling problem. They perform a relatively limited exploration of the search space and generally produce good quality solutions within reasonable computing time. Modifications are made to the original model in this component. The adaptations provide the iterative and interactive nature of the model. If there are additional requests from customers, it allows the scheduler to run the component any time in order to build an improved model.

\section{B. Hot rolling scheduling editor}

Hot rolling scheduling editor is the most important part of the decision support system, which decide directly scheduling horizon. In order to utilize the automated scheduler more efficiently, the component provides most of the schedule manipulation functions. When the scheduler makes turns, Data collection system should gather the necessary data through Data access platform. The decision support system can generate automatically multiple turns for a shift. Then hot rolling scheduling editor also allows schedulers to edit manually outputs by User interface. Hot rolling scheduling editor was programmed to execute within a Windows environment with dialog boxes, menus, drawer button, command buttons and quickly pop-up menus similar to those which are familiar to the majority of computer users, as illustrated in Figure 3. The "EDA_PR Algorithm" button employs the algorithms to make the schedule. The system displays the violation information using the different color schemes. This display with different color aims to simplify the system and emphasize the flexible use. Schedulers can edit turns conveniently according to the information. 


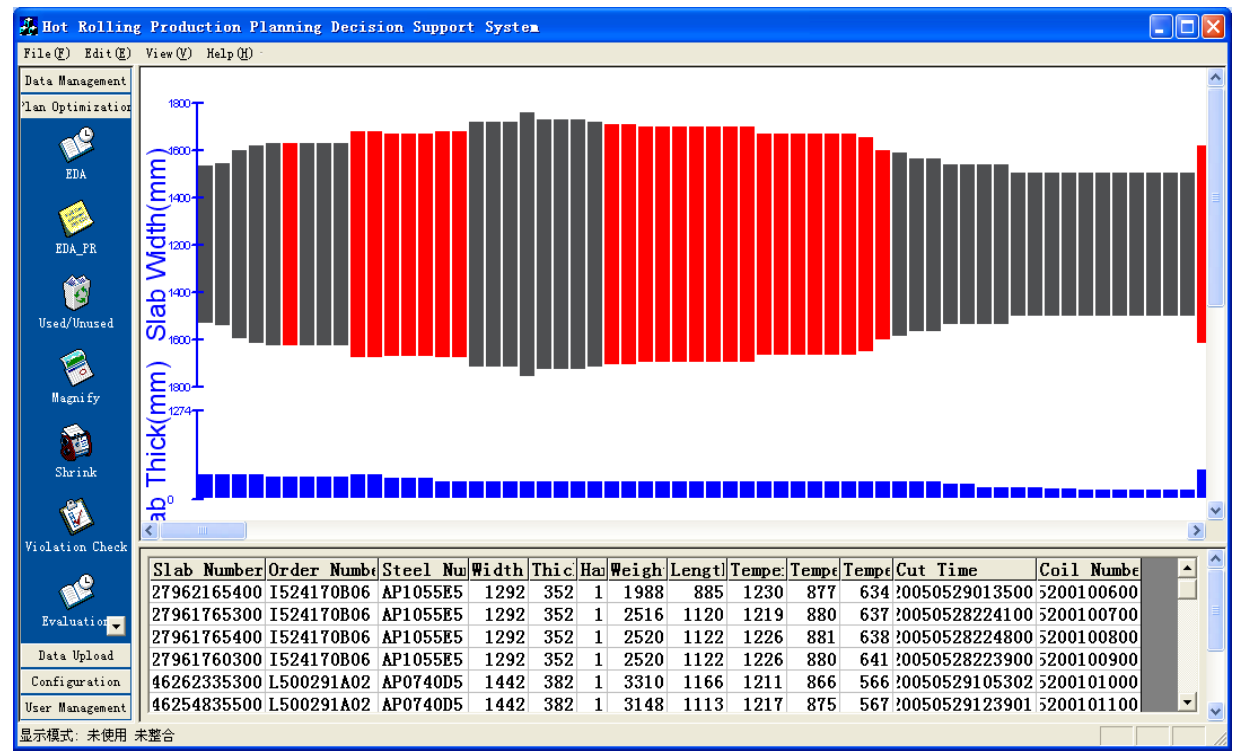

Figure 3. The graph show of hot rolling sequence generated by EDA_PR.

\section{CONCLUSIONS}

In this paper, we have studied the hot rolling scheduling problem, which is derived from the actual steel production, and some features make the solution methodology more difficult. Therefore, the EDA_PR algorithm is proposed in this paper, which is to hybridize EDA and PR with heuristics to solve the hot rolling scheduling problem. The method has both the advantages of EDA, the ability to find the higher performance solutions, and that of PR, the ability to conduct fine-tuning in the quality of solutions and to find better solutions. Meanwhile, PR is embedded into EDA as neighborhood search to improve solutions. The computational experiments for problem instances have shown that the hybrid algorithm has more potential for improvement to solve the hot rolling scheduling problem compared with the manual scheduling method. Finally, a decision support system for the hot rolling scheduling is designed to improve the existing scheduling process. The hybrid algorithm has been embedded in the decision support system, and the performance of the system has been tested using various scheduling scenarios. The most popular features of the system are the graphical interface and architecture of component management so that the decision support system is convenient enough to be applied to other steel companies.

\section{ACKNOWLEDGMENT}

This research is supported by Scientific Research Foundation of Liaoning Educational Committee (Grant No. L2010196).

\section{REFERENCES}

[1] L. Lopez, M.W. Carter, and M. Gendreau, "The hot strip mill production scheduling problem: a tabu search approach," European Journal of Operational Research 106 (1998) 317-335.

[2] P. Cowling, "Optimisation in steel hot rolling," Optimization in industry, Wiley, Chichester, England, 1995, PP. 55-66.

[3] P. Cowling and W. Rezig, "Integration of continuous caster and hot strip mill planning for steel production," Journal of Scheduling 3 (2000) $185-208$.

[4] E. Balas, "The prize collecting traveling salesman problem," Networks 19 (1989) 621-636.

[5] L.X. Tang, J.Y. Liu, A.Y. Rong, and Z.H. Yang, "Multiple traveling salesman problem model for hot scheduling in Shanghai Baoshan Iron \& Steel Complex," European Journal of Operational Research 124 (2000) 267-282.

[6] M. Pelikan, D.E. Goldberg, and F. Lobo, "A survey of optimization by building and using probabilistic models," Computational Optimization and Applications 21 (2002) 5-20.

[7] M. Hauschild, M. Pelikan, "An introduction and survey of estimation of distribution algorithms," Swarm and Evolutionary Computation 1 (2011) 111-128.

[8] F. Glover, M. Laguna, and M. Marti, " Fundamentals of Scatter Search and Path Relinking," Control and Cybernetics 39(3) (2000) 653-684.

[9] S.C. Ho, M.Gendreau, " Path relinking for the vehicle routing problem," Journal of Heuristics 12 (2006) 55-72. 